\title{
A NOTE ON CERTAIN GENERALIZED FREE PRODUCTS
}

\section{ROBERT J. GREGORAC}

Let $A \pi B \mid U$ be the generalized free product of $A$ and $B$ with amalgamated subgroup $U$ [2]. Recall that a group $G$ is residually $P$ for some group property $P$, if, for any element $g \in G$ not the identity, there exists a normal subgroup $N$ not containing $g$ such that $G / N$ has property $P$.

Baumslag [1] has shown that if $A$ and $B$ are finitely generated torsion free nilpotent groups and $U$ is cyclic, then $A \pi B \mid U$ is residually finite. However, it can be verified that $G=g p\left(a, b \mid a^{2}=b^{3}\right)$ is a generalized free product of the above type which is not residually a finite $p$-group for any prime $p$. Hence we might ask what possible finite indices normal subgroups in $A \pi B \mid U$ might have.

The following theorem, which grew out of a conversation with Thomas Head, shows that normal subgroups of all possible finite indices exist in $A \pi B \mid U$, when $U$ is cyclic.

Theorem. Let $A$ and $B$ be finitely generated nontrivial torsion-free nilpotent groups and let $U$ be cyclic. Then $S=A \pi B \mid U$ has an infinite cyclic factor group.

Proof. Case 1. Suppose $A$ and $B$ are cyclic, say $S=g p\left(a, b \mid a^{h}=b^{k}\right)$. Then the abelianization of $S, S / S^{\prime}$, is an infinite abelian group and so $S / S^{\prime}$ has an infinite cyclic factor group. Hence so does $S$.

Case 2. Let $A$ and $B$ both be abelian groups, but not both cyclic. Let $H$ and $K$ be the isolators of $U$ in $A$ and $B$, respectively. The groups $H$ and $K$ are direct factors of $A$ and $B$, respectively (see [2], isolated subgroups), say $A=C \times H, B=D \times K$. Either $C \neq 1$ or $D \neq 1$, say $C \neq 1$. The natural epimorphisms of $A$ onto $C$ and $B$ onto 1 can be extended to an epimorphism of $S$ on to $C$, because the amalgamated subgroup $U$ maps on to the trivial group in $C$. $C$ is a finitely generated torsion-free abelian group, so $C$ has an infinite cyclic factor group, and so must $S$.

Case 3. Now suppose the nilpotent classes of $A$ and $B$ are $n$ and $m$ $(>0)$, respectively. Let $U=\langle u\rangle$ and suppose either $u \in Z_{n-1}(A)$ or $u \in Z_{m-1}(B)$, where $Z_{n-1}(A)$ is the last proper term of the upper central series of $A$. Say $u \in Z_{n-1}(A)$. In this case we can extend the natural epimorphisms $A \rightarrow A / Z_{n-1}(A)$ and $B \rightarrow 1$ to an epimorphism of $S$ onto the torsion-free abelian group $A / Z_{n-1}(A)$, for the amalgamated subgroup $U$ maps onto the identity.

Received by the editors July 19, 1966. 
Finally, suppose $u \notin Z_{n-1}(A)$ and $u \notin Z_{m-1}(B)$. Since $A / Z_{n-1}(A)$ and $B / Z_{m-1}(B)$ are torsion free $U \cap Z_{n-1}(A)=1=U \cap Z_{m-1}(B)$. Hence there is an epimorphism of $S$ onto $G=A / Z_{n-1}(A) \pi B / Z_{m-1}(B) \mid U$, and $G$, by either Case 1 or Case 2 , has an infinite cyclic factor group. This completes the proof.

Corollary. Let $A$ and $B$ be groups with normal subgroups $N$ and $M$, respectively, such that $A / N$ and $B / M$ are finitely generated nontrivial torsion-free nilpotent groups. Then $S=A \pi B \mid U, U$ cyclic, has an infinite cyclic factor group.

Proof. Let $U=(u)$, and suppose either $u \in N$ or $u \in M$. Say $u \in N$. Then we can extend the epimorphisms $A \rightarrow A / N$ and $B \rightarrow 1$, to an epimorphism of $S$ onto $A / N$. Hence $S$ has an infinite cyclic factor group.

If $u \notin N$ and $u \notin M$, then since $A / N$ and $B / M$ are torsion free, $U \cap N=1=U \cap M$. Hence we can find a natural epimorphism of $S$ onto $A / N \pi B / M \mid U$, and the result follows by the theorem.

This corollary says that if $A$ and $B$ have infinite cyclic factor groups, so does $A \pi B \mid U$. This probably is the main result here, but our proofs of the cases considered suggest possible noncyclic homomorphic images.

We mention a final special case.

Corollary. Let $A$ and $B$ be free groups. If $U$ is cyclic, then $S$ $=A \pi B \mid U$ has an infinite cyclic factor group.

\section{REFERENCES}

1. G. Baumslag, On the residual finiteness of generalized free products of nilpotent groups, Trans. Amer. Math. Soc. 106 (1963), 193-209.

2. A. Kurosh, The theory of groups, Vol. II, Chelsea, New York, 1960.

Iowa State University 